\title{
La movilidad como estrategia para la regeneración urbana desde el espacio público: un taller de urbanismo en Zaragoza.
}

\author{
Raimundo Bambó-Naya1, Pablo de la Cal'2, Isabel Ezquerra ${ }^{3}$, \\ Sergio García-Pérez ${ }^{4}$, Javier Monclús ${ }^{5}$ \\ U.P. Arquitectura, Universidad de Zaragoza, Zaragoza, España \\ E-mail: ${ }^{1}$ rbambo@unizar.es, ${ }^{2}$ pdelacal@unizar.es, \\ 3iezquerra@unizar.es, ${ }^{4}$ sgarciap@unizar.es, ${ }^{5}$ jmonclus@unizar.es
}

\begin{abstract}
Resumen. Frente a los años en los que la expansión de la ciudad ha protagonizado la principal preocupación de la práctica profesional, la agenda urbana reciente exige volver la mirada hacia la ciudad existente. Los talleres de urbanismo que se desarrollan en los últimos años de carrera tienen también la responsabilidad de afrontar este reto. Esta comunicación presenta una experiencia de carácter docente e investigador en la que se aborda la regeneración urbana de un barrio vulnerable en el marco del Máster en Arquitectura de la Universidad de Zaragoza. El barrio zaragozano de Torrero, desde el punto de vista socioeconómico, presenta procesos de envejecimiento y aparición de enclaves muy localizados con concentración de familias de bajos recursos. Además, el barrio posee condiciones morfológicas especificas, como su posición de borde, su aislamiento respecto al resto de la ciudad, la colindancia con un extenso pinar o la heterogeneidad de sus tejidos urbanos, que han generado procesos de transformación particulares. Tras una fase de análisis y diagnóstico operativo, se apostó por una estrategia de intervención a escala de barrio que considera la movilidad como instrumento eficaz para recualificar sus espacios públicos y corregir desigualdades espaciales y sociales entre sus distintas áreas. A partir de la adaptación de los conceptos y experiencias de las supermanzanas, el taller definió una serie de actuaciones concretas, comprobando en distintas situaciones y escalas las posibilidades que esta estrategia brinda para la mejora del barrio. Todo ello con el objetivo de avanzar en la construcción de una ciudad más compleja, diversa, segura e inclusiva.
\end{abstract}

Palabras clave: barrio vulnerable, periferia, supermanzana, movilidad urbana, docencia, transferencia de conocimiento.

\section{Introducción}

El protagonismo de la circulación es un hecho reconocido en el urbanismo del siglo XX. La sistematización de las redes y sistemas viarios ha sido fundamental tanto en la ordenación del crecimiento urbano como en las reformas interiores de las ciudades contemporáneas (Bambó-Naya y MonclúsFraga 2019). La tradición anglosajona de la ciudad jardín y el suburbio jardín utilizó la jerarquización viaria y la reorganización de los equipamientos básicos como herramientas para la creación de unidades residenciales de grandes dimensiones, buscando mejorar la calidad del espacio público y la recuperación del espíritu de vecindad (Perry 1929). Con la imposición de las visiones más funcionalistas (Benevolo, Melograni, y Giura Longo 2000), especialmente a partir de la Carta de Atenas (Le Corbusier, Giradoux, y de Villeneuve 1943) $\mathrm{y}$, sobre todo, con el incremento exponencial de la circulación rodada y la proliferación de vehículos desde la década de los sesenta del pasado siglo, la pérdida de calidad del espacio público fue un resultado común a casi todas las 
ciudades (Mumford 2000). Las respuestas a ese proceso oscilaron entre la adaptación del tejido urbano a las necesidades del automóvil y las tentativas de control mediante la redefinición de las mallas viarias tradicionales con estrategias basadas en la delimitación de áreas pacificadas. El importante informe de Colin Buchanan, El tráfico en las ciudades (1963), recomendaba la potenciación de una red viaria principal para favorecer el tráfico rodado; pero también la delimitación de 'áreas ambientales' en las que los peatones y la calidad ambiental disfrutaran de clara prioridad. Más de 50 años después la idea se ha recuperado en forma de 'supermanzanas', como sectores libres de todo tráfico de paso, en los que se consideran prioritarias las mejoras medioambientales y la estrategia de mejora de los espacios públicos (Rueda 2017; Bodego, Lavadinho, y Lensel 2018; Pérez Igualada 2017).

En el taller de urbanismo del Máster en Arquitectura de la Escuela de Ingeniería y Arquitectura de la Universidad de Zaragoza (2018-19) se ha abordado la regeneración de un barrio periférico de la ciudad de Zaragoza a partir de esas estrategias de mejora de los espacios públicos basada en la reordenación del sistema de movilidad interna y externa, mediante la redefinición de las jerarquías viarias y la introducción de supermanzanas. A continuación se expone la situación del barrio, el proceso seguido en el taller y las conclusiones del trabajo desarrollado con alumnos, vecinos y administración.

\section{EI taller de Proyectos Urbanos y Paisajísticos Integrados}

El taller de urbanismo del Máster en Arquitectura acumula seis años de experiencia trabajando en barrios vulnerables de la ciudad de Zaragoza (España) (Berruete, de la Cal, y García 2014; de la Cal y García 2015; de la Cal y Bambó 2016; Bambó, de la Cal, y García-Pérez 2017, 2018). En esta ocasión, el taller focaliza en el barrio de Torrero-La Paz, donde el marco de desarrollo y las condiciones específicas de la zona han posibilitado la

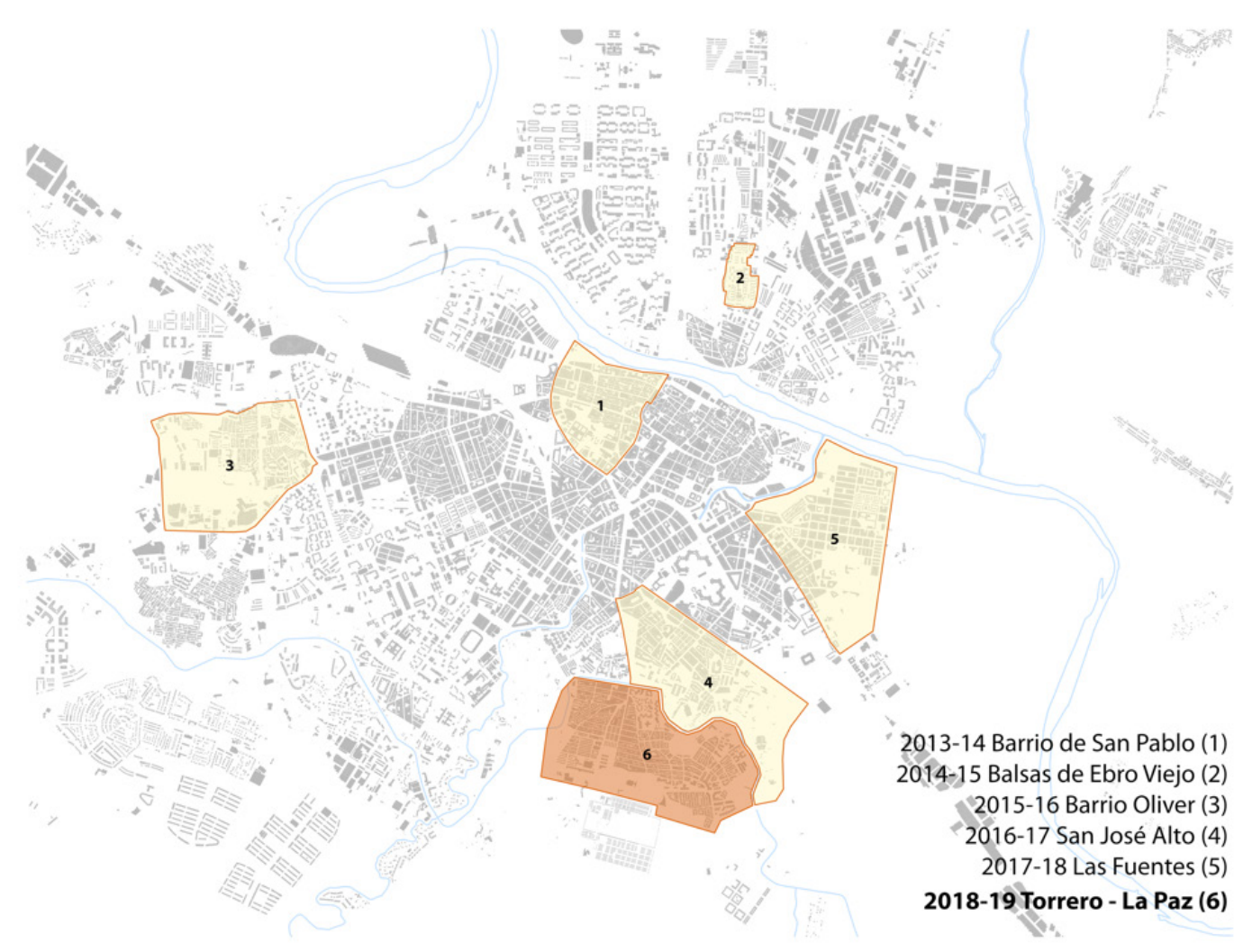

Fig. 1. Ámbitos de trabajo en la edición 2019 y en las anteriores del taller de urbanismo del Máster en Arquitectura de la Universidad de Zaragoza. (Fuente: elaboración propia, 2019) 
opción de otorgar una importancia estratégica a la movilidad urbana (Fig.1).

Situado al sur de la ciudad, el barrio de TorreroLa Paz nace ligado a la construcción del Canal Imperial. Hasta el primer tercio del siglo XX fue considerada una zona privilegiada de la ciudad, en la que las parcelaciones situadas sobre la orilla del Canal acogían viviendas destinadas a la segunda residencia de la incipiente burguesía zaragozana que encontraba aquí su zona de recreo. La ubicación del cementerio municipal al sur del barrio desde 1834 y su buena conexión con el centro de la ciudad a través del tranvía desde finales del siglo XIX fueron factores que favorecieron el interés urbano de esta zona, reforzado por actuaciones como las repoblaciones forestales llevadas a cabo en la segunda década del siglo XX. Esta conexión con la ciudad estaba reducida históricamente al Puente de América que suponía el paso sobre el Canal del antiguo Camino de Torrero (actual Paseo Cuéllar), para continuar su camino hasta el Cementerio por la vía que hoy conocemos como Avenida América. Sin embargo, el traslado de la cárcel de la calle Predicadores a la Avenida de América en 1928, supuso un cambio en la tendencia del barrio. Aquel desarrollo de la zona denominada Barrio de Venecia, que acogía a la clase burguesa, se vio frenado y el barrio se transformó en una zona residencial de trabajadores, receptora de población obrera llegada con el éxodo rural. Este crecimiento estaría caracterizado, sobre todo en el sector de La Paz, por la autoconstrucción de 'casetas', de una o dos plantas con patio trasero, sobre unas parcelaciones particulares adaptadas a unas situaciones preexistentes sin atender a ningún plan de ordenación. Los planes generales de la segunda mitad del siglo XX recogieron como oficiales estas situaciones, legalizando así muchas parcelaciones hasta entonces alegales, $\mathrm{y}$ establecieron reservas para dotaciones públicas en los márgenes del barrio.

Desde el plan de 1957 se permitió la densificación de las parcelas, hecho que ha condicionado el heterogéneo desarrollo del estado de transformación del tejido original del barrio, aumentando la presión edificatoria sobre el espacio libre que además se ha visto invadido por el vehículo privado. Del mismo modo que el barrio encuentra su límite norte en el Canal, aparecen al sur otras infraestructuras naturales y viarias que dibujan otro límite más "difuso". El Tercer Cinturón, que cruza el barrio de Este a Oeste, podría entenderse como el límite de la ciudad compacta, teniendo una función eminentemente urbana. Los Pinares de Venecia y el Cuarto Cinturón son los otros dos importantes elementos infraestructurales que se ubican al sur (Garrido Palacios (dir.) y Faci Olmos 2003; Adiego et al. 1984).

En este contexto, el taller comenzó con la realización por parte de los alumnos de un análisis integrado. Para ello, los equipos focalizaron su análisis a través de la mirada reflejada en distintos textos, agrupados en temáticas que han permitido adoptar una visión 'amplia', capaz de revelar las oportunidades específicas de un barrio aparentemente consolidado. Han sido claves las lecturas sobre la forma física de la ciudad a través de los ojos de D. Mangin (2004) y M. Solá-Morales (1987), junto a los retos actuales -o no tan actuales-que debe afrontar la movilidad urbana (Buchanan 1973; Alexander 1968; Rueda 2017), sobre todo en relación con el espacio público (Cullen 1974; Lynch 1985; Gehl 2014; Gehl, Kaefer, y Reigstad 2006) y las oportunidades que el urbanismo paisajístico le ofrece (L. Mumford s. f.; Corner 2009). Al conocer la composición social del barrio, bastante envejecida y con alto porcentaje de población extranjera, fue interesante profundizar en la relación entre el soporte físico y las desigualdades sociales que en él se producen (Hayden 1995; Secchi 2015). El crecimiento del barrio al margen de la planificación de la ciudad, condicionaba la reflexión que el papel del planeamiento puede jugar en la evolución del mismo (de Terán 2018; Portas 2003). Más allá del interés de cada una de las reflexiones individuales realizadas por cada uno de los equipos, la experiencia más enriquecedora del taller fue la que surgió tras la puesta en común de todas las perspectivas analizadas. Así se pudo garantizar un nivel de profundidad adecuado junto a una visión integrada y transversal, capaz de abordar la problemática compleja a la que debe hacer frente el barrio.

El análisis realizado ha permitido obtener un diagnóstico operativo capaz de identificar no solo los problemas más importantes, 


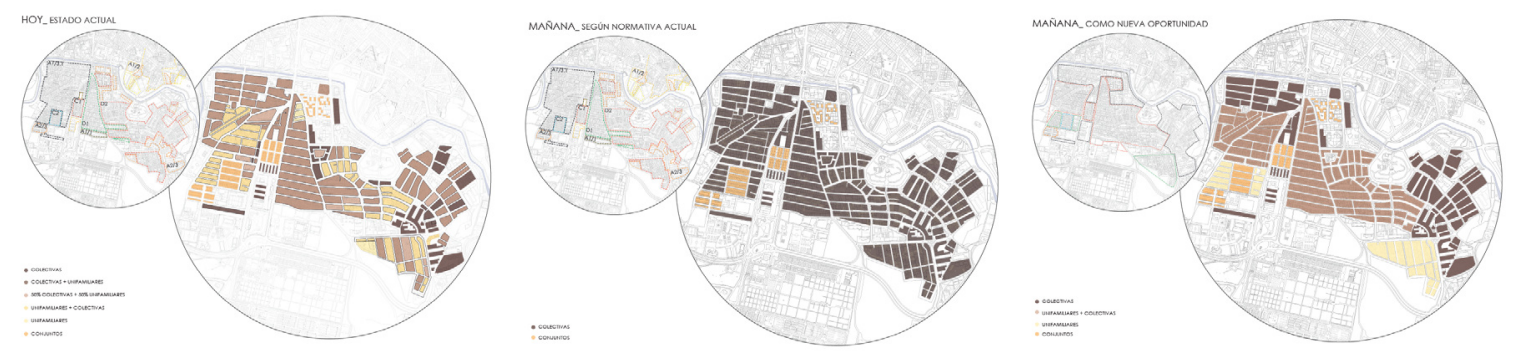

Fig. 2. Análisis del Plan General de Ordenación Urbana vigente. Escenario actual, escenario futuro acorde al PGOU y alternativa posible. (Fuente: Rodrigo Anchelergues, Silvia Gómez, Sara Molinero y Clara Mur, 2019)

sino también las oportunidades latentes en el barrio. Entre los primeros destaca la congestión del sistema viario del barrio, muy condicionado por las conexiones históricas. Además, sorprende comprobar cómo algunos de los crecimientos recientes, más que paliar los efectos visibles de esta situación, no hacen sino incrementarlos, no favoreciendo la continuidad entre tejidos urbanos. Tampoco las reformas urbanas desarrolladas recientemente en el interior del barrio, como la llevada a cabo en la plaza de la Memoria Histórica en el solar que ocupó la cárcel de Torrero, han sabido aprovechar la oportunidad de generar nuevos espacios públicos de referencia, cuyo uso es a día de hoy limitado por problemas de ordenación y diseño. Además, la legalización de las situaciones al margen del planeamiento se ha visto ineficaz en el transcurso del tiempo, al dar por bueno un sistema viario insuficiente para las nuevas demandas de estacionamiento, el transporte público y el desarrollo de un sistema de espacios públicos de calidad; situación más preocupante si se tiene en cuenta la posibilidad de densificación que todavía permite el planeamiento vigente. También desde un punto de vista patrimonial, este posible proceso produciría una pérdida de identidad del tejido urbano, a favor de una excesiva homogeneización del mismo (Fig.2)

Sin embargo, las especificidades del barrio de Torrero-La Paz ofrecen, al mismo tiempo, grandes oportunidades. Su posición elevada con respecto a la ciudad y los elementos paisajísticos que lo rodean, el Pinar de Venecia y el Canal Imperial, son un gran potencial a desarrollar. La puesta en marcha en el año 2002 del Tercer Cinturón, podría permitir la jerarquización del sistema viario de la zona, favoreciendo la eliminación del tráfico de paso y dando solución a uno de los principales problemas que presenta el barrio (calles estrechas, falta de aparcamientos, escasez de espacios públicos de referencia, ineficacia de las líneas de transporte público, etc.). Diversas fortalezas y oportunidades para los que el interés de la iniciativa privada, podría suponer una oportunidad de catalizar procesos integrales de mayor alcance. Estas propuestas se han tratado de desarrollar en la segunda fase del taller.

\section{Proceso interactivo movilidad-diseño de espacio público}

El diagnóstico del conjunto Torrero-la Paz nos presenta un punto de partida con premisas nítidas. El Canal Imperial de Aragón sigue siendo hoy una barrera urbana más que una conexión con la ciudad, y los puentes o puntos de conexión con los barrios colindantes son hoy escasos. De hecho, el puente de América, y su eje de prolongación dentro del barrio, la avenida de América, es un punto de congestión. Torrero queda estructurado en torno a este eje, complementado con la calle Fray Julián Garcés, mientras que la Paz presenta una estructura mucho más caótica, a veces solo comprensible para los vecinos habituados a un sistema de calles sinuosas trazadas en una topografía quebrada. Y en los bordes del Sur, el Tercer Cinturón ha dejado una prudente distancia con el tejido urbano, en la que permanecen retazos de pinares, cada vez más aislados y descontextualizados, del Monte de Torrero. El impulso adquirido por los desarrollos del Sur (Parque Venecia, Puerto Venecia, etc.) se deja notar en la revitalización reciente del sector de la calle Zafiro. Pero en el interior de Torrero y la Paz, los espacios públicos tienen un tráfico 
creciente y una escena urbana completamente dominada por el vehículo estacionado, en un escenario que será agravado por las condiciones previstas en el Plan General de Ordenación Urbana, con una densificación edificatoria prevista y aún no completada.

El conjunto es resultado, por lo general, de un sistema de parcelaciones particulares, que ha producido un sistema espacial y construido muy homogéneo y sin cualificar. Por ello, tanto desde el punto de vista funcional que atiende a la movilidad interior y a las conexiones con el resto de la ciudad, como desde el punto de vista espacial, se hace necesario establecer una nueva jerarquía en el espacio público. La implementación de un sistema de 'supermanzanas' se vislumbra como una estrategia acertada en este caso, ya que permite reorganizar los flujos de circulación peatonal y rodada, con un planteamiento que debe atender tanto a 'lo pequeño' (las plazas estanciales, los recorridos peatonales, y las sendas cicables), como a 'lo grande' (las conexiones transversales entre Torrero y La Paz y la creación de un sistema de tres ejes Norte-Sur capaces de descongestionar el eje de la avenida de América y de reorganizar el transporte público de una manera más eficiente) (Reinoso, Abarca, y Osuna 2015).
Desde el primer momento se ha visto claro que estas medidas pueden llevarse a cabo si se encuentra una alternativa adecuada al estacionamiento del vehículo privado en las calles del barrio. La apuesta por calles peatonales, la decisión de introducir transporte colectivo en determinados itinerarios, o la creación de nuevas plazas son actuaciones que requieren eliminar o disminuir de manera muy sensible las dotaciones actuales de aparcamientos en la vía pública. Por ello, durante todo el ejercicio del Taller, un Equipo de Movilidad (formado por Karim Benchiha y Marina Sanz) se ha dedicado a trabajar con distintos escenarios, en función de la posibilidad de creación de nuevas 'bolsas de aparcamiento público' en el área de influencia de cada una de las 'supermanzanas' propuestas (Fig. 3).

Al mismo tiempo, los sistemas espaciales y de equipamientos adquieren mayor dimensión transformadora si se consideran desde una óptica general. Para ello un Equipo Director (formado por Rodrigo Anchelergues, Silvia Gómez, Sara Molinero y Clara Mur) garantiza la coherencia entre las piezas que se desarrollan a nivel 'proyecto urbano' por el resto de equipos, siempre desde el compromiso estrecho con los 'escenarios de movilidad'.

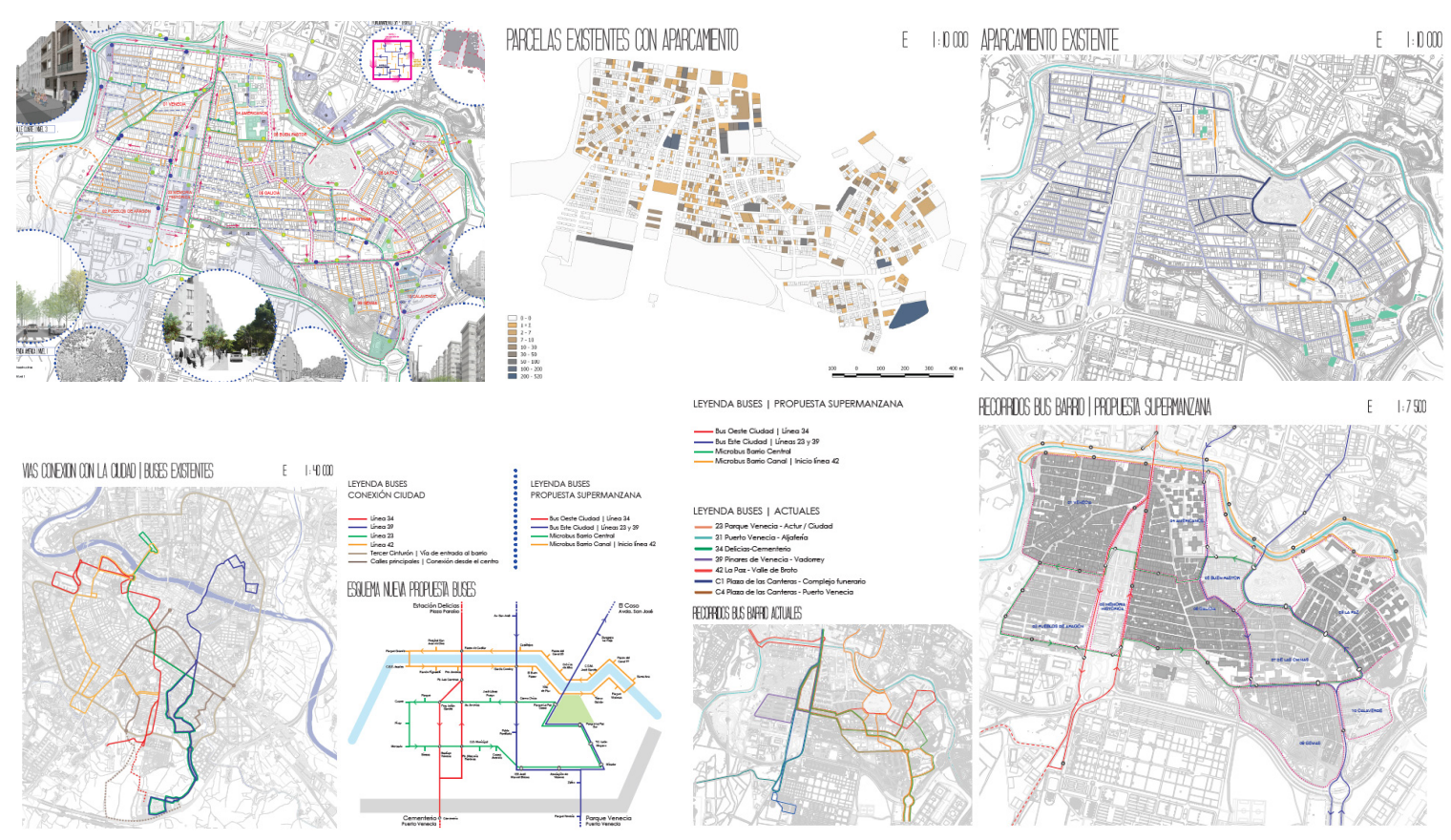

Fig. 3. Análisis y propuestas para la movilidad de Torrero-La Paz. (Fuente: Karim Benchiha y Marina Sanz, 2019) 
La coordinación transversal entre el Equipo Director, el Equipo de Movilidad y los diferentes Equipos de 'proyecto urbano' que trabajan en distintas piezas o ámbitos del barrio, ha sido quizá el aspecto más interesante del Taller. En un barrio de dimensiones físicas tan exiguas y de parámetros de espacio público tan apretados, las decisiones proyectuales en los ámbitos pequeños condicionan en gran medida algunas medidas relativas a la reordenación de la movilidad interior o al planteamiento de algunas líneas estratégicas, y viceversa. Así, las soluciones finales de 'diseño urbano' han sido ratificadas y consolidadas cuando se ha comprobado que encajan a la perfección en la dimensión general del barrio, y de manera especial en el nuevo escenario de movilidad urbana sostenible que se trata de definir (Fig. 4).

Este es el caso, por ejemplo, del diseño para una nueva avenida que podría descongestionar la actual condición de eje omnipresente que posee la avenida América. La propuesta para una nueva vía urbana que discurre por el Oeste del barrio, entre el puente del Canal Imperial y la glorieta del Tercer Cinturón, una vez supera el nivel de esquema general de movilidad, afronta la escala de proyecto urbano. Y se plantea cuestiones que en su conjunto permiten superar el rechazo inicial que este planteamiento tiene por su afección a la masa de pinares. Hacer un nuevo eje Oeste (Equipo Álvaro Giménez, Rodrigo Miranda y Elisa Pottetti) es hacer que el espacio filtro o de encuentro 'barrio-pinar' tenga más funciones urbanas: el planteamiento de traslado del Colegio en la esquina noroeste del barrio permite reforzar de manera clara la relación con el Canal Imperial, el tráfico rodado atraviesa un sistema de sinuosas trazas dentro del pinar, mientras que la fachada urbana se consolida como un sistema de paseo urbano, reforzado por los nuevos espacios dotacionales $\mathrm{y}$ comerciales.

Desde un planteamiento estratégico se ha tratado de consolidar dos nuevos ejes o itinerarios en sentido transversal TorreroLa Paz. Por una parte, el 'eje interior norte', que recorre los espacios de la supermanzana 'Venecia' y trata de extender el efecto de la plaza de las Canteras hacia el Este, conectando con el Centro Cívico Torrero y con el parque de la Paz. En ocasiones, se debe recurrir a estrategias de permeabilización de la trama urbana actual y creación de nuevas replacetas $\mathrm{y}$ espacios peatonales (Equipo Manuel Mayor y Elena Comeras), y en otras ocasiones se trata de potenciar la presencia de algunos equipamientos ya existentes en espacios que superen la condición actual de 'calle' para pasar a ser una 'plaza equipada' (Equipo Daniel de Buen y Alberto Ibáñez). Por otra parte, el 'eje interior sur', que parte del nuevo desarrollo comercial que se plantea en las antiguas instalaciones del colegio Lestonnac con un doble formato conjunto, mercado de barrio y mediana superficie, así como un Centro de

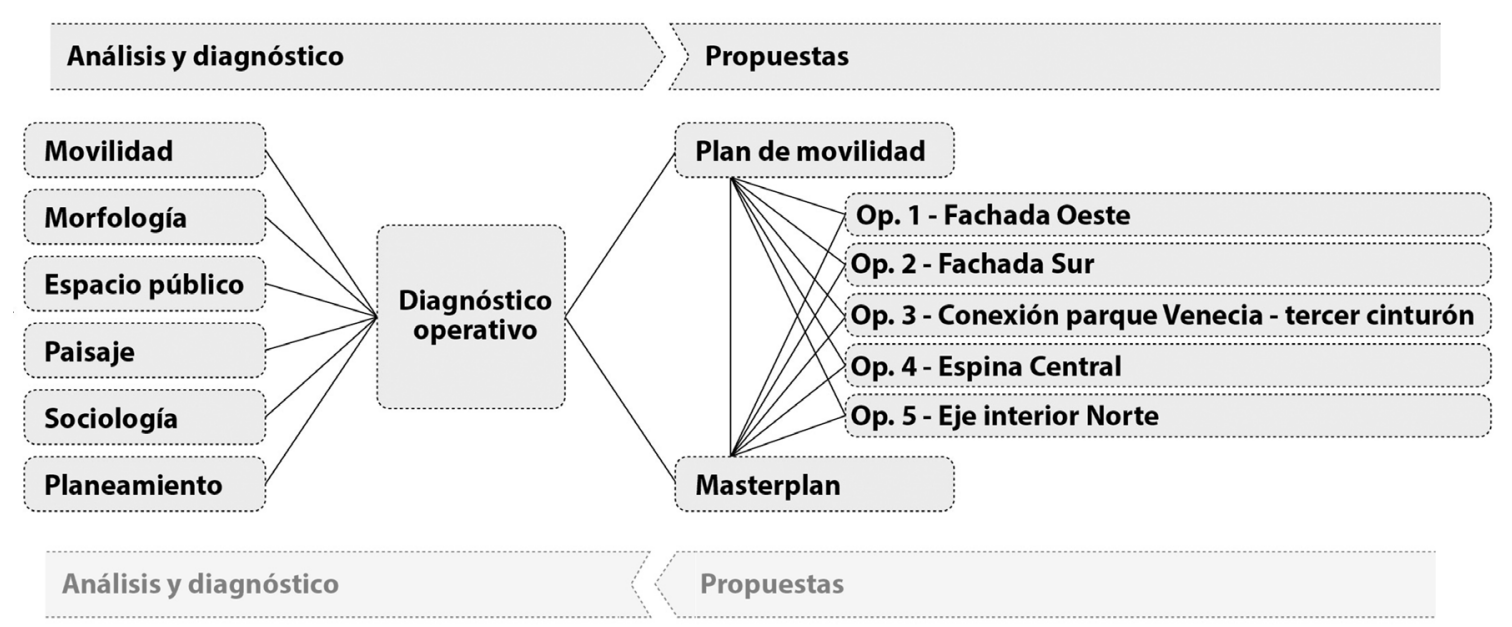

Fig. 4. Proceso interactivo movilidad-espacio público. (Fuente: elaboración propia, 2019) 


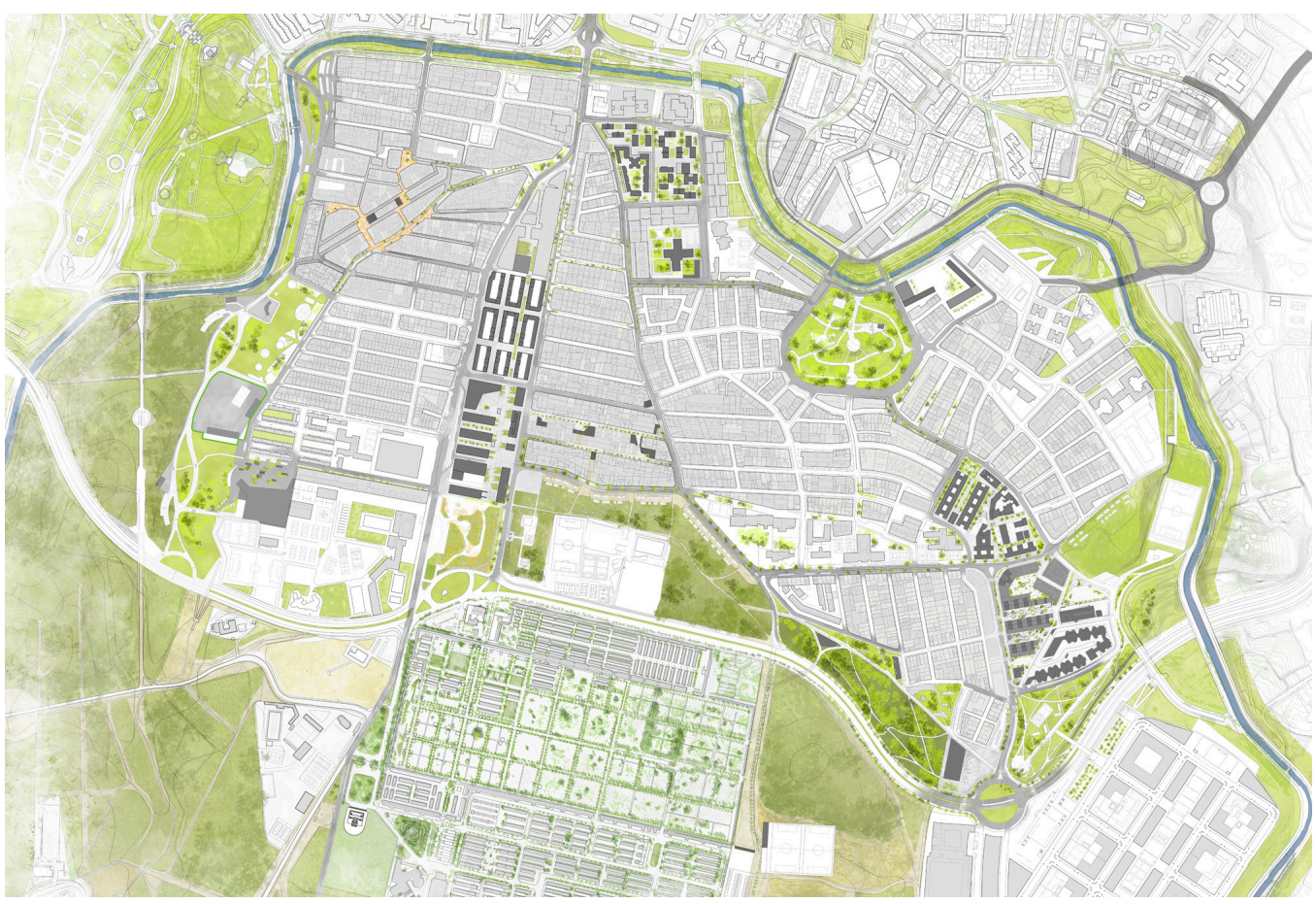

Fig. 5. Plan Director de Torrero - La Paz, con el conjunto de propuestas elaboradas en el Taller. (Fuente: Rodrigo Anchelergues, Silvia Gómez, Sara Molinero y Clara Mur, a partir del trabajo del conjunto de los alumnos, 2019)

Interpretación de los pinares de Torrero y la estepa zaragozana (Equipo Alejandro Perales, Carlos Puértolas y Adán Molinos). Este eje atraviesa el barrio por el sur, cosiendo traseras y resolviendo el encuentro con los pinares hasta llegar al estratégico enclave del cabezo, junto al acueducto del Canal Imperial sobre el Tercer Cinturón (Equipo Laura Martínez y Andrea Simón).

Los trabajos generales han detectado el efecto beneficioso que para el extremo suroeste de La Paz está teniendo el desarrollo del Tercer Cinturón y la actividad generada en los barrios del Sur (Sede de la Policía Local en Parque Venecia, nuevas dotaciones en Parque Venecia, Puerto Venecia, etc.). Estas tensiones urbanas generan ciertos problemas de congestión puntual del tráfico y requieren propuestas de renovación urbana en estos ámbitos. Espacios que antes eran solares residuales pueden ser objeto de recualificación, y la visión conjunta de una 'supermanzana' ayuda para que las actuaciones adquieran un sentido completo, como el planteamiento integral que se hace en la manzana situada al este de la calle Zafiro (Equipo Carlos Beltrán y Rosa Caballero).
Asimismo, la propuesta que analiza el final de la calle Venecia y la conexión con la fachada Este del Cementerio (Equipo Arturo Cebollero y Diego Ibáñez), que puede consolidarse como un espacio de nueva centralidad también se enmarca en esta estrategia general de mejorar la relación del barrio con los espacios del sur.

Actuaciones de regeneración urbana en ámbitos muy específicos también resultan necesarias cuando se interviene en conjuntos urbanos que poseen características singulares. Es el caso del denominado 'Conjunto de los americanos' en el que se ha realizado una interesante propuesta de renovación ambiciosa, que plantea la construcción de un aparcamiento subterráneo conjunto en una posición central, la creación de un eje transversal de mayor vitalidad urbana y la decidida incorporación de los patios interiores como 'espacios comunitarios de calidad' (Equipo Helena del Río y Gemma Bel). Se trabaja con las zonas privadas, introduciendo jerarquía urbana y definición espacial en un espacio que carece de estos atributos.

Se han propuesto interesantes actuaciones de esponjamiento de la trama urbana, trabajando 
desde la oportunidad que ofrece la condición actual de determinados solares vacíos. Así, se ha intervenido en zonas muy densas y que requieren una decidida renovación, como el sistema de calles de la calle Lugo y adyacentes (Equipo Laura Cabeza y Pilar García), o la propuesta en el entorno de la 'plaza de las Chinas' (Equipo Jorge Moreno y Henry Córdova), donde en un espacio ciertamente recluido y mal conectado con la malla principal se intenta introducir continuidad comercial, desde un tratamiento arquitectónico de transformación espacial. Y así se ha hecho también en la revisión de las condiciones actuales de la plaza de la Memoria (Equipo Paloma Góez e Inés Villarroya) y en su relación con el conjunto residencial Fray Julián Garcés (Fig.5).

En todas estas actuaciones de 'proyecto urbano' las decisiones sobre las posibilidades de eliminar plazas de estacionamiento privado en la vía pública, reorganizar los sentidos de circulación, establecer diferentes grados de peatonalización, ubicar con criterios de intermodalidad eficiente los itinerarios y las paradas de las líneas de transporte público, etc. han sido los ingredientes de un ejercicio constante de 'interacción escalar' en el proyecto de regeneración urbana.

\section{Conclusiones}

En cualquier barrio vulnerable, se debe proceder con un planteamiento que abarque una doble escala de trabajo. Por un lado, conectividad exterior y conexión con la ciudad. Y, por otro lado, movilidad interior y regeneración del espacio público. Esta doble escala de trabajo debe ser continua en todo el proceso de diseño urbano, donde los aspectos de movilidad y los de regeneración de los espacios de los barrios tienen una retroalimentación mutua y permanente. La organización de un Taller de regeneración urbana con una estructura de un Equipo Director, un Equipo de Movilidad y distintos Equipos de proyectos urbanos ha resultado muy acertada (Bambó-Naya et al. 2018).

Los planteamientos de regeneración, apoyados de manera muy directa en propuestas de revisión integral de la movilidad en el barrio, desde la atención permanente a la calidad del espacio público, son muy adecuados para superar la mera apuesta por una 'mejora de lo existente' y avanzar hacia una transformación real del barrio, que permita la evolución de Torrero-La Paz hacia escenarios de mayor equidad, diversidad y eficiencia (Monclús 2017).

Las propuestas que se muestran tienen esa doble virtud. Por un lado, están tuteladas bajo una óptica general que garantiza un funcionamiento del conjunto del barrio, mejor relacionado con el resto de la ciudad, con un funcionamiento más eficiente en su estructuración interior, que plantea un mallado de avenidas estructurantes con la intención de superar el histórico 'cuello de botella' de la avenida de América y su puente sobre el Canal Imperial. Y, al mismo tiempo, una propuesta atenta a la calidad espacial de los ámbitos más duros y densos del interior, con actuaciones de regeneración urbana en los enclaves más vulnerables, con nuevas 'venas verdes' en el interior del barrio, que superan la actual uniformidad y evolucionan hacia un sistema de espacios diversos que introduce calidad ambiental en el interior del barrio.

\section{Agradecimientos}

Este trabajo se ha desarrollado en el marco del convenio de transferencia OTRI 2019/0218, financiado por Zaragoza Vivienda, y de los proyectos de investigación predoctoral financiados por el Ministerio de Economía y Competitividad (BES 2015/072536) y el Ministerio de Educación, Cultura y Deporte (FPU 2016/06737). En particular, los autores quieren expresar su agradecimiento a los vecinos que nos han acompañado durante esta experiencia y, en especial, a todos los alumnos participantes en el Taller.

\section{Referencias}

Adiego, Elvira, José Antonio Baguena, José Luis Calvo, Enrique Grillo, Carlos Guelbenzu, Victor Guelvenzu, José Félez, María José Moreno, y Ana Taboada. 1984. Zaragoza: Barrio a Barrio. Vol. 3. Editado por Federación de Asociaciones de Vecinos. Zaragoza: Cometa S.A. 
Alexander, Christopher. 1968. «La ciudad no es un árbol [A city is not a tree, 1965]». Cuadernos summa - nueva visión: enciclopedia de la arquitectura de hoy 9: 20-30. https://sistemasdeproyecto.files. wordpress.com/2012/10/alexander-c-unaciudad-no-es-un-c3a1rbol.pdf.

Bambó-Naya, Raimundo, Pablo de la CalNicolás, Carmen Díez-Medina, Sergio García-Pérez, y Javier Monclús-Fraga. 2018. «From theory to practice: five years of urban regeneration workshops». Journal of Technology and Science Education 8 (3): 179-91. https://doi.org/10.3926/jotse.382.

Bambó-Naya, Raimundo, y Javier MonclúsFraga. 2019. «De la manzana a la supermanzana. Recuperación e innovación en la cultura urbanística». En La casa. Espacios domésticos, modos de habitar. II Congreso Internacional cultura y ciudad. Granada, 23-25 enero 2019, editado por Juan Calatrava Escobar, 531-43. Granada: Abada.

Bambó, Raimundo, Pablo de la Cal, y Sergio García-Pérez. 2017. «Taller San José Alto. La memoria de un barrio como motor de regeneración urbana / San José Alto workshop. The memory of a district as the driving force for urban regeneration». En Regeneración Urbana (IV). Propuestas para el barrio de San José / Urban regeneration (IV). Proposals for San José Neighborhood, editado por Javier Monclús y Raimundo Bambó, 82-95. Zaragoza: Prensas de la Universidad de Zaragoza - Ayuntamiento de Zaragoza.

- 2018. "Crecimiento en el borde y renovación interior: vasos comunicantes para la regeneración de Las Fuentes (Zaragoza)». En Regeneración urbana (V). Propuestas para el barrio de Las Fuentes, editado por Javier Monclús y Raimundo Bambó, 8497. Zaragoza: Prensas de la Universidad de Zaragoza. https://doi.org/10.26754/ uz.9788417633363.

Benevolo, Leonardo, Carlo Melograni, y Tommaso Giura Longo. 2000. La proyectacióndelaciudadmoderna. Traducido por Carlos Gómez González. Barcelona: Gustavo Gili. http://search.ebscohost.com/ login. aspx ?direct $=$ true $\& d b=$ cat $00574 a \& A$
$\mathrm{N}=$ cbzara.b1548568\&site $=$ eds-live.

Berruete, Francisco, Pablo de la Cal, y Miriam García. 2014. «Taller San Pablo-Zgz. Una experiencia académica de regeneración urbana». En Regeneración urbana. Propuestas para el barrio de San Pablo, Zaragoza / Urban Regeneration. Proposals for San Pablo Neighborhood, Zaragoza, editado por Javier Monclús (dir.), Ricardo S. Lampreave, y Carmen Díez Medina, $120-$ 29. Zaragoza: Prensas de la Universidad de Zaragoza - Ayuntamiento de Zaragoza.

Bodego, Béatrice, Sonia Lavadinho, y Bernard Lensel. 2018. «Des trames urbaines aux 'supermanzanas'». Urbanisme, n.o 409: 1726.

Buchanan, Colin. 1963. Traffic in Towns: A Study of the Long Term Problems of Traffic in Urban Areas. London: Her Majesty's Stationery Office. https://books.google.es/ books?id=-OUjCQAAQBAJ\&printsec $=$ fro ntcover\&hl=es\&source $=$ gbs_ge_summary $\mathrm{r} \& \mathrm{cad}=0 \# \mathrm{v}=$ onepage $\& \mathrm{q} \& \mathrm{f}=$ false . . 1973. El tráfico de las ciudades [Traffic in Towns, 1963]. Madrid: Tecnos.

Corbusier, Le, Jean Giradoux, y Jeanne de Villeneuve. 1943. La charte d'Athènes. Plon. Paris.

Corner, James. 2009. «Terra Fluxus [Terra Fluxus, 2006]». En Naturaleza y artificio: el ideal pintoresco en la arquitectura y el paisajismo contemporáneos, editado por Iñaki Ábalos, 133-48. Barcelona: Gustavo Gili.

Cullen, Gordon. 1974. El paisaje urbano. Tratado de estética urbanística [The Concise Townscape, 1961]. Barcelona: Blume.

Garrido Palacios (dir.), José, y Yolanda Faci Olmos. 2003. Evolución histórica y socioeconómica del barrio de Torrero-La Paz (Zaragoza). Zaragoza: Geodesma.

Gehl, Jan. 2014. Ciudades para la gente [Cities for People, 2010]. Traducido por Juan Décima. Buenos Aires: Infinito. http://www. urbangateway.org/system/files/documents/ publicspace/cities_for_people-_spanish_ final_ss2.pdf.

Gehl, Jan, Lotte Johansen Kaefer, y Solvejg Reigstad. 2006. "Close encounters with buildings». Urban Design International 11 (1): 29-47. https://doi.org/10.1057/palgrave. 
udi.9000162.

Hayden, Dolores. 1995. The power of place : urban landscapes as public history. MIT Press.

la Cal, Pablo de, y Raimundo Bambó. 2016. «Taller Barrio Oliver-Zgz. Urbanismo para combatir la vulnerabilidad urbana». En Regeneración urbana (III). Propuestas para Barrio Oliver, Zaragoza, editado por Javier Monclús y Raimundo Bambó. Zaragoza: Ayuntamiento de Zaragoza - Prensas de la Universidad de Zaragoza.

la Cal, Pablo de, y Miriam García. 2015. "¿Qué queremos decir cuando hablamos de regenerar Balsas de Ebro Viejo en Zaragoza?» En Regeneración urbana (II). Propuestas para el polígono Balsas de Ebro Viejo. Zaragoza, editado por Javier Monclús, Carlos Labarta, y Carmen Díez Medina, 11135. Zaragoza: Sociedad Municipal Zaragoza Vivienda / Prensas de la Universidad de Zaragoza.

Lynch, Kevin. 1985. La buena forma de la ciudad [Good city form, 1981]. Barcelona: Gustavo Gili.

Mangin, David. 2004. «La ciudad sectorizada. Y Dios creó el automóvil...» En La ville franchisée - Formes et structures de la ville contemporaine, traducido por PUPC y Javier Monclús. Paris: Editions de la Villette.

Monclús, Javier. 2017. «Urban regeneration and urban design strategies». En 24th ISUF International Conference. Valencia: Universitat Politècnica de València.

—. Javier. 2018. "Urban Transport and Technological Urbanism". En Urban Visions, editado por Carmen Díez Medina y Javier Monclús, 187-96. Cham: Springer International Publishing. https://doi. org/10.1007/978-3-319-59047-9_18.

Mumford, Eric. 2000. The CIAM Discourse on Urbanism, 1928-1960. Cambridge: M.I.T. Press.

Mumford, Lewis. s. f. «The Neighborhood and the Neighborhood Unit». The Town Planning Review. Liverpool University Press. Accedido 20 de septiembre de 2018. https://doi.org/10.2307/40101548.

Pérez Igualada, Javier. 2017. «La idea de supermanzana en los polígonos de viviendas de Valencia (1956-1971)». ZARCH, n.o 8 (octubre): 132-43. https://doi.org/10.26754/ ojs zarch/zarch.201782151.

Perry, Clarence Arthur. 1929. «The neighborhood unit. A scheme of arrangement for the family-life community». En Regional study of New York and its environs, Volume VII, Neighborhood and Community Planning, Monograph 1. New York: Regional Plan of New York and its Environs.

Portas, Nuno. 2003. «El surgimiento del proyecto urbano [L'emergenza del progetto urbano, 1998]». Traducido por Javier Monclús. Perspectivas Urbanas / Urban perspectives 3: 1-11. http://upcommons. upc.edu/bitstream/handle/2099/555/art03-2. pdf? sequence $=1 \&$ isAllowed $=y$.

Pozueta (dir.), Julio, Francisco José Lamíquiz, y Mateus Porto. 2009. La ciudad paseable: recomendaciones para la consideración de los peatones en el planeamiento, el diseño urbano y la arquitectura. Madrid: CEDEX.

Reinoso, Rafael, Javier Abarca, y Fernando Osuna. 2015. «Superblocks. Resiliencia, capacidad de adaptación y resistencia a la obsolescencia». En Obsolescencia y Regeneración: Viviendas del siglo XX en el nuevo milenio, editado por AA.VV. Sevilla: Universidad de Sevilla.

Rueda, Salvador. 2017. «Supermanzanas. Nueva célula urbana para la construcción de un nuevo modelo funcional y urbanístico de Barcelona». En Jaca: ciudadpaisaje / landscapecity, editado por Carlos Ávila y Pablo de la Cal, 52-79. Zaragoza: Prensas de la Universidad de Zaragoza.

Secchi, Bernardo. 2015. La ciudad de los ricos y la ciudad de los pobres [La città dei ricchi e la città dei poveri, 2013]. Madrid: Catarata.

Solà-Morales Rubió, Manuel de. 1987. «La segunda historia del proyecto urbano». UR: urbanismo revista, n.o 5: 21-27.

Terán, Fernando de. 2018. Antes de salir por la puerta del tiempo. Visión personal de un urbanismo real. Madrid: Lampreave. 